\title{
The Fault Diagnosis of Shield Disc Cutter Based on Neural Network

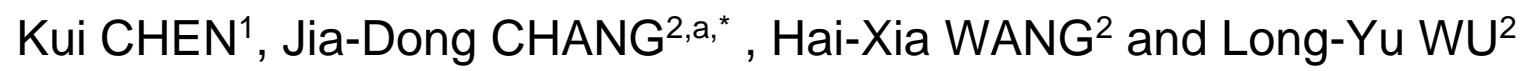

\author{
${ }^{1}$ State key laboratory of shield machine and boring technology, \\ Henan, Zhengzhou, 450001 \\ ${ }^{2}$ Luoyang institute of science and technology, \\ Henan, Luoyang, 471023 \\ achangjiadong4228865@126.com
}

Keywords: Neural network, Shield machine, Disc cutter wear, Fault diagnosis.

\begin{abstract}
In order to predict the wear fault of disc cutter in shield machine, a BP neural network model was established and then trained by inputting several groups of disc cutter wear fault samples data including cutter rotate speed, cutter head torque, tunnelling speed, total thrust force and soil pressure force into the model. Through testing, the model has a good agreement with the practice, and can realize on-line monitoring and prediction for the fault of cutter wear.
\end{abstract}

\section{Introduction}

With the development of underground engineering, shield plays more important roles in the construction fields such as urban subway, mountain tunnel and drainage pipeline. During the shield tunnelling, the wear of the cutter effects directly on the shield tunnelling efficiency. The wear of disc cutter is the main wear of the whole cutters. If the disc cutter wear develops to a certain extent, the disc cutter must be changed by new ones, otherwise it will aggravate the other cutters wear and then form a vicious cycle. Hence, it is essential to conduct the diagnosis and prediction of the disc cutter wear accurately. To judge the disc cutter is wear or not, the conventional methods are opening the soil chamber to inspect, using sensors of the cutter wear and analysis of the tunnelling parameters, etc[1-2]. There will be high risk in opening the soil chamber to check the disc cutter, and it may cause the excavation surface collapse or affect the safety of the surrounding building. The sensors can be installed on minor cutters. The method using analysing the tested tunnelling parameters is conducted based on the establishment of the relative relationship among the parameters including the total press force, total torque and tunnelling speed. The paper utilized the sample data of the tunnelling parameters to establish the regression prediction model of the disc cutter wear, based on the nonlinear formula among the rotate speed of cutter, tunnelling speed, total press force and disk torque [3]. A Tunnel Boring machine (TBM) on-line cutter wear monitoring system was designed based on the eddy current sensor, which converted the changes of distance between the sensor and the cutter ring before and after it was worn to the voltage signal, and then the actual cutter ring wear data were got through signal conversion [4-6]. A new method for forecasting the wearing of disc-cutters using Elman neural network model is proposed [7], the disc-cutters wearing is forecasted according to the difference between the predicted excavation speed from the model and the actual excavation speed.

The research above showed that there is interrelate between the tunnelling parameters and the cutter wear fault, so it is available to predict the cutter fault by using the tunnelling parameters to build the regression model, but the method has bad adaptability of different geological conditions.

Shield itself has the detection function of the tunnelling parameters online, such as disk torque, rotate speed, soil pressure of chamber, total press force, the main voltage and electricity, etc. These parameters data contains the information of the cutter wear. Meanwhile, the BP (Back Propagation) neural network has stronger capability of the nonlinear function mapping, it can approximate any continuous function with arbitrary precision. Therefore, the tunnelling parameters are taken as input to establish the BP neural network of disc cutter fault diagnosis is feasible. 


\section{BP Neural Network of Disc Cutter Wear Fault Diagnosis}

BP neural network is a studying network of multilayer forward feedback model, which is composed of input layer, hidden layer and output layer. It is an efficient and practical classification and recognition tool based on error back propagation method of studying and training [8]. A BP neural network with three layers can be used to approximate any continuous function and map the nonlinear relationship between the input layer and output layer. The three layers of BP neural network including input layer, hidden layer and output layer can reduce the workload of calculation and avoid the complexity of network structure. In the learning process, it makes the output of neural network gradually approach the target output process for the connections weights between the modified input layer and the hidden layer, and the hidden layer and the output layer, and bias value.

When disc cutter wear to lose efficiency, cutter disk rotational speed, torque, promoting speed, total thrust force of cutter disk and chamber pressure from shield machine can change significantly, so a BP neural network of a hidden layer is used in fault diagnosis of shield disc cutters wear of forward feedback BP neural network used has, and it is shown in Figure 1. The input layer includes five neural network units, they respectively correspond rotational speed of cutter disk, cutter head torque, promoting speed, total thrusting force and soil pressure force. The output layer has a neural unit in correspondence with disc cutter wear.

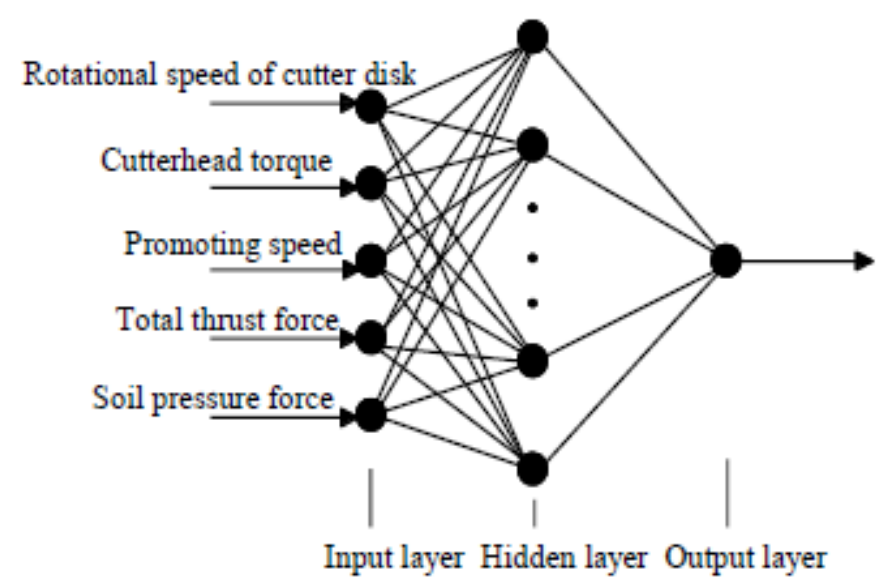

Fig. 1. Topological structure of BP neural network from fault diagnosis of disc cutter wear

The steepest descent method is used in BP neural network of learning rule, which the error square value of the network continues to decrease using the back propagation to continuously modify the weights and thresholds of the network connection. The learning and training process includes signal forward transmission and error back propagation. Signal forward transmission is the input layer signal pass through the hidden layer, and the hidden layer is transmitted to the output layer, the signal is processed by every layer, and neuron state of each layer affects the state of the next layer. By training the neural network from data samples, when a group of known data is input, correspondingly a set of known and expected data is output. If the output value is less than the expected output value, the output error signal is returned in the reverse propagation process, and the output error is reduced continuously by modifying the connection weights between the layers and the threshold value of each layer.

BP neural network model of the cutter wear failure is that data eigenvalue of the engineering sample is extracted as the network input, and the network is mapped to the weights of the network, that the network is trained to learn, and the network encountered a new set of driving parameters data input, that the model and the existing model of the characteristics of the existing model is compared, so the wear results is determined. BP network has the characteristics of parallel computing, self-learning, adaptive and associative memory, which can be identified as the sample pattern in the training study. 


\section{Simulation and Calculation of the BP Neural Network}

The simulation flow chart of the shield cutter wear's network diagnosis is shown in Figure 2.

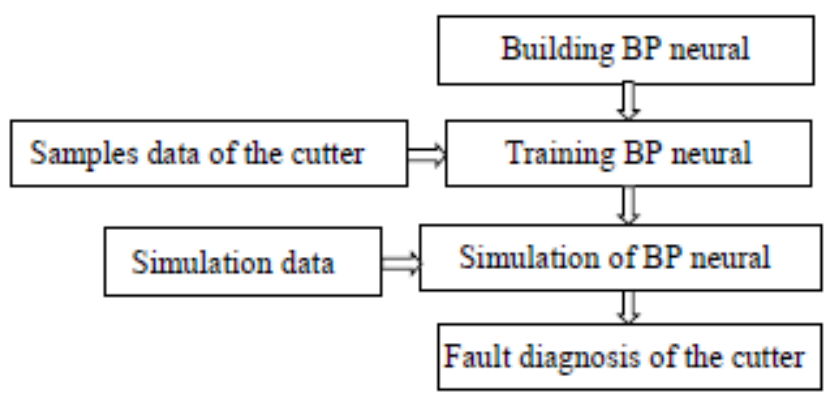

Fig. 2. The fault diagnosis flow chart of the shield cutter

The BP neural network can be set up, trained and simulated by the embedded programs in the NNbox, a neural network toolbox in MATLAB [9].

Using the special function 'newff( )' to establish the BP neural network, it's form is:

net=newff(P,T, S,TF, BTF, BLF, PF, IPF,OPF,DDF)

Where, P- RxQ1 matrix of Q1 representative R-element input vectors;T- SN×Q2 matrix of Q2 representative SN-element target vectors; $\mathrm{Si}$ - sizes of $\mathrm{N}-1$ hidden layers, $\mathrm{S} 1$ to $\mathrm{S}(\mathrm{N}-1)$; TFi- transfer function of it's layer; BTF- backprop network training function; BLF - backprop weight/bias learning function; PF- performance function; IPF- row cell array of input processing functions; OPF- row cell array of output processing functions; DDF- data division function.

The training of the neural network is implemented through calling the 'train' function, in the form of

[NET,ep,Y,E,Pf,Af]=train(net,P,T,Pi,Ai)

The Train function calls the net.trainParam function to carry on the training of the neural network. In the input variable of the function, net is the network object, $\mathrm{P}$ indicates the network input, usually the sample value vector or matrix, $\mathrm{T}$ denotes the target vector, Pi represents the initial state of the input delay and $\mathrm{Ai}$ is the initial state of the layer delay. In the return value of the function, NET denotes a new network; ep represents the training record; Y expresses the output of the network, and $\mathrm{E}$ is the error between the output and the target vector; Pf shows the training of the termination of the input delay state and Af indicates the training time of the termination of the termination of the state.

The learning of BP neural network is realized by loading the network learning function. The learngd function is a gradient descent learning function. By changing the input and error of neurons and the learning rate of weights and threshold, the weight or threshold rate are calculated.

The simulation of the well learned neural network is the core of fault diagnosis, which can be realized by calling ' $\operatorname{sim}($ )' function. The form is used as the following:

[Y,Pf,Af,E,perf] $=\operatorname{sim}($ net,P,Pi,Ai,T)

Where, net- neural network object; P- network input; Pi- input delay state; Ai- initial state of the layer delay; T- target vector; Y- network output; Pf- training of the termination of the input delay state; Af- training of the termination of the time delay state; E- output and error between the target vector; perf- network performance index.

By identifying the simulation results, the fault result is outputted. If the disc cutter wear achieve the critical extent, there is need to replace the disc cutter by a new one, then the result is outputted as 1 , otherwise the output of the neural network is set as 0 .

\section{Example of the Disc Cutter Fault Diagnosis on BP Neural Network}

The normalized processing of the disc cutter fault parameters is conducted according to the formula (1), Table 1 shows the fault sample data after normalized processing. 


$$
x_{i}=\frac{x_{i}-x_{\min }}{x_{\max }-x_{\min }}
$$

Table 1. Normalization sample data of disc cutter fault characteristic parameters

\begin{tabular}{|c|c|c|c|c|}
\hline $\begin{array}{c}\text { Cutter rotate } \\
\text { speed }\end{array}$ & $\begin{array}{c}\text { Cutterhead } \\
\text { torque }\end{array}$ & $\begin{array}{c}\text { Promoting } \\
\text { speed }\end{array}$ & $\begin{array}{c}\text { Total thrust } \\
\text { force }\end{array}$ & $\begin{array}{c}\text { Soil pressure } \\
\text { force }\end{array}$ \\
\hline 0.96883 & 0.37768 & 0.00092 & 0.11040 & 0.01922 \\
\hline 1 & 0.39425 & 0.00246 & 0.20048 & 0.01922 \\
\hline 0.98337 & 0.60528 & 0.00369 & 0.26673 & 0.01922 \\
\hline 0.94713 & 0.72124 & 0.00401 & 0.34994 & 0.02145 \\
\hline 0.91481 & 0.72250 & 0.00401 & 0.38307 & 0.01922 \\
\hline 0.87273 & 0.84507 & 0.00246 & 0.41933 & 0.01922 \\
\hline 0.98701 & 0.98893 & 0.00246 & 0.41983 & 0.02145 \\
\hline 0.97714 & 0.88801 & 0.00092 & 0.41981 & 0.02145 \\
\hline 0.96416 & 0.78924 & 0 & 0.41234 & 0.02145 \\
\hline$\ldots$ & $\ldots$ & $\ldots$ & $\ldots$ & $\ldots$ \\
\hline 0.89714 & 0.73842 & 0.00030 & 0.40898 & 0.01922 \\
\hline
\end{tabular}

The BP neural network program of the disc cutter fault diagnosis were completed in Matlab according to the part 3 above. The Graphical User Interface (GUI) was developed. GUI is convenient in the operation process of the system, and it makes the cumbersome process calls simplified. All the functions are programmed into a subfile (.m), and the user can achieve the diagnosis result by inputting the test samples.

Selecting the part of the data in Table 1 as the training sample data to train the BP neural netwrok of the disc cutter fault diagnosis. The another part of the data in Table 1 as fault diagnosis sample data were inputted in the BP neural network which had been trained successfully. The diagnosis interface and the result are shown in Figure3.

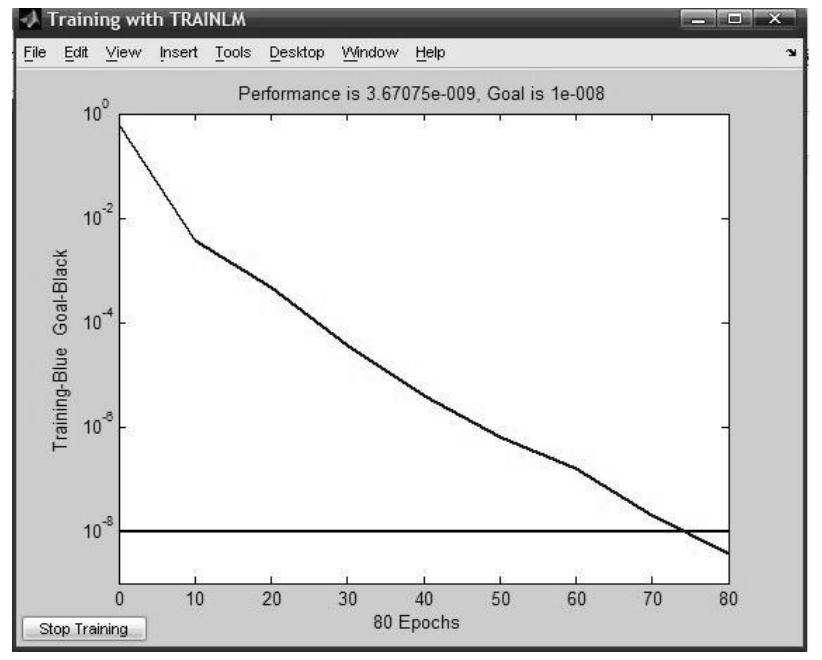

(a) Training performance

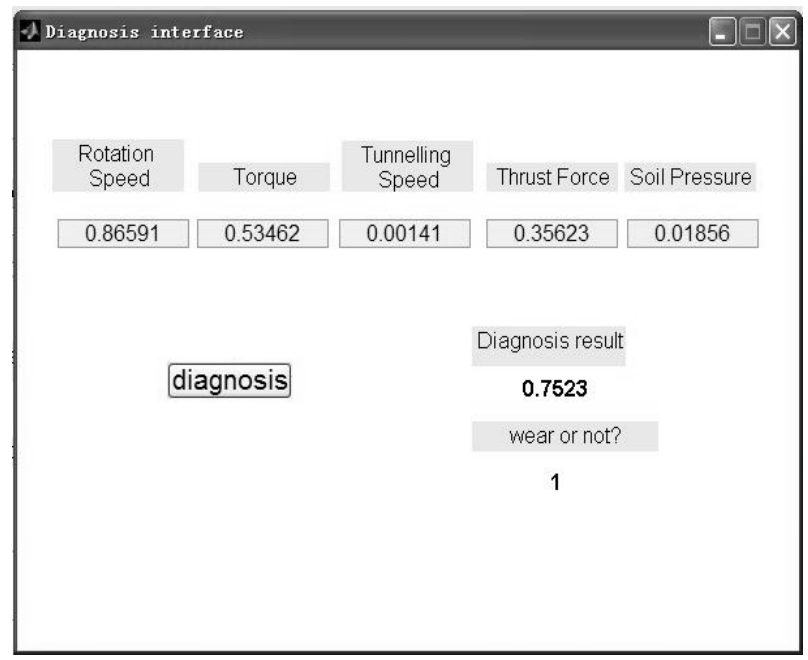

(b)Diagnosis interface

Fig. 3. Fault diagnosis interface of the shield disc cutter

\section{Conclusions}

In the process of shield tunnelling, if the cutter wear fault could not be found in time and it is replaced, the working conditions of other disc cutter would be deteriorated, the fast wear and failure of the cutter would be caused, which could cause the economic loss and reduce construction efficiency. In 
construction process, judge disc cutter wear failure mainly rely on the operator's experience, and according to the changes of the shield tunnelling parameters, judge disc cutter wear failure and whether to replace the disc cutter, or shield construction in a certain time interval open check tool, when the geological condition is not stable need pressure inspection and replacement of cutting tools, artificial judgment depends on experience of the operator, so the error rate is high. In fact, if there is one or more cutter wear failure, it will affect the change of shield driving parameters, which the parameters of shield tunnelling parameters include disc cutter wear failure information, in this paper a BP neural network model will be presented basing on the BP neural network. The accuracy of the results is verified by computer simulation. Graphical user interface (GUI) system is more convenient for the user's operation, which is based on the Windows environment in the Matlab program of support development. The theoretical support is provided for the online diagnosis system of cutter wear in the process of shield construction, a new way is developed for reducing the construction risk and improving the construction efficiency.

\section{Acknowledgements}

The research was financially supported by the National Basic Research Program 863 of China, the subject number is 2012AA041803. And the research was also financially supported by the Opening Program of State Key Laboratory of Shield Machine and Boring Technology, the subject number is 2013-08.

\section{References}

1. H. Zhang. Study on the method of estimating cutter wear of shield in mix-face ground[C]. Proceeding of Shanghai's international symposium on tunnelling. (2005), pp.461-471

2. H. Zhao. The Research of Shield Disc Cutter Wear Detection Technology [D]. STDU,(2015)

3. X. Li, X. Su. Forecast of wear shield's disc cutters based on multivariate nonlinear regression [J]. J. LNTU (Natural Science), 2(2009).pp.281-283

4. W. Zheng, H. Zhao, H. Lan. Design of On-line Monitoring System for Tunnel Boring Machine's Disc Cutter Wear [J]. Instrument Tech. \& Sensor, 2 (2015), pp.46-50.

5. Y. Wu. Research on Disc Cutter Wear Prediction Model of Tunnel Boring Machine in Complex Groud[D]. Northeasten University,(2013)

6. X. Zhao. Abrasion monitoring System of the disc cutters on shields [D]. HAUST, (2014)

7. X. Li, X. Su. A new method for forecasting shield's disc-cutters wearing based on Elman neural network [J]. J. LNTU (Natural Science), 29,6 (2010), pp.1121-1124

8. J. Wei, F. Chen, M. Jiang. Application of BP neural network in fault diagnosis of radar device [J]. Mod. Elect. Tech., 35,19(2012), pp.131-134

9. Y. SHI. Realization of BP Neural Network Based on Matlab[J]. J. XNU, 31,5(2010), pp.86-88

10. Y. Fu. Application Research of wavelet neural network technology in the shield machine fault diagnosis [D]. Zhengzhou University,(2014)

11. G. CHEN, D. XING. Shield machine fault prediction based on least square method and BP neural network [J]. Construction Mechanization, 04 (2013), pp.65-67 